\title{
Recent progress of immunology research in China
}

\author{
LENG FangWei \\ Institute of Biophysics, Chinese Academy of Sciences, Beijing 100101, China
}

Received July 10, 2011; accepted August 21, 2011

Citation: Leng F W. Recent progress of immunology research in China. Sci China Life Sci, 2011, 54: 1068-1070, doi: 10.1007/s11427-011-4248-9

To celebrate the 60th anniversary of SCIENCE CHINA, the second issue of Science China Life Sciences (2010) focuses on immunology, with six articles contributed by immunologists working both in and outside China. The articles review the current position and recent developments in the field of immunology, including tumor necrosis factor (TNF) receptor-associated factor (TRAF) family molecules, innate recognition, plasmacytoid dendritic cells (pDCs), costimulatory molecules, T cells, and humanized mouse models.

As early as 1798, the word "vaccination" was coined specifically to describe the injection of smallpox vaccine. In 1880, Louis Pasteur published a paper on Pasteurella septica cholera, heralding the beginning of a new scientific field: Immunology. From then on, Pasteur's germ theory of human infectious disease facilitated the development of various vaccines, leading to a greater understanding of the immune system which, in turn, resulted in the expansion of the field of Immunology. For a century, immunologists around the world have enhanced our understanding of the immune system; from humoral and cellular adaptive immunity to the pattern recognition involved in innate immunity. Alongside great advances due to the expansion of molecular biology over the past decade, Immunology has become a central pillar of the life sciences, developing many branches such as Medical Immunology, Tumor Immunology, Clinical Immunology and Transplantation Immunology. As an integral component of medical science, Immunology plays an important role in deepening the understanding of the pathophysiology of human disease, and also contributes to the development of modern therapeutics to treat infection,

email: fangweileng@126.com autoimmune diseases, allergies and cancer. In recent years, Chinese immunologists have produced many important studies in Immunology. The following reviews provide a brief overview of recent advances in Immunology, highlighting the contributions of leading Chinese immunologists.

The first review "TRAF-mediated regulation of immune and inflammatory responses" by Wang et al. [1] focuses on the TRAF family, beginning with a comprehensive introduction to structure and function. Over the past few years, research studies have established a central role of TRAF proteins in inflammation and immunity. The TRAF family plays divergent and non-redundant roles in regulating the many signal transduction pathways involved in innate and adaptive immune responses. These molecules participate in signal transduction by a large number of receptor families, including the TNF receptor family and the Toll-like receptor-interleukin-1 receptor family. The review summarizes the divergent roles played by TRAF proteins in positive and negative regulation of the canonical and non-canonical $\mathrm{NF}-\kappa \mathrm{B}$ pathways and type I interferon pathways, and also points out many aspects of TRAF biology that, although not clear, may still be of great significance to related research.

The second review is entitled "On self-non-self discrimination in pattern recognition" by Liu et al. [2]. The first section of this article discusses the development of immunity from a historical perspective. The second part deals with two different patterns of innate immune recognition. The third part then goes on to summarize the latest advances in the authors' own research into the CD24-SiglecG/10 pathway. According to their studies, the CD24/Siglec 10 pathway discriminates pathogen-associated molecular patterns 
from danger-associated molecular patterns, enabling a cross-reactive pattern recognition pathway to initiate immunity against pathogens without significant immunemediated self-destruction to the tissues $[3,4]$. Although recent studies have greatly enhanced our understanding of the CD24/Siglec 10 pathway, there are still several challenging questions that remain to be answered.

pDCs are unique and essential immune cells capable of producing large amounts of type I interferons in response to viral infection [5,7]. The third review entitled "Plasmacytoid dendritic cells in antiviral immunity and autoimmunity" by Tang et al. [8] focuses on the molecular mechanisms underlying pDC activity in antiviral immunity and autoimmunity. The review summarizes the remarkable progress made over the past 10 years in understanding pDC biology, and provides a detailed introduction to pDC biology, including their discovery, characterization, and function in antiviral immunity and autoimmunity. Because of the importance of pDCs to antiviral immunity and autoimmunity $[9,10]$, future research will lead to novel therapeutic approaches to the induction of human antiviral immunity and the treatment of autoimmune diseases.

The next review entitled "Structural immunology of costimulatory and coinhibitory molecules" by Wang ShengDian and Chen LiePing [11] focuses on the $\mathrm{T}$ cell costimulatory pathways that play a critical role in regulating the immune response. In recent years, several co-signaling pathways have been identified [12,13]. An explosion of research into costimulation has resulted in incredible growth in the understanding of the molecular structures involved, and has provided the basis for further understanding of $\mathrm{T}$ cell costimulatory pathways and the subsequent development of therapeutic interventions. This review describes in detail the structure of co-signaling molecules in the context of their function and therapeutic application. Predictably, as research progresses, gradually increasing understanding of the structure of costimulatory and co-inhibitory molecules will provide further impetus to the study of the biological function of costimulatory pathways, and encourage their therapeutic application.

The current understanding is that $\mathrm{T}$ cells activate innate immune cells to clear pathogens. Surprisingly, it has become evident that $\mathrm{T}$ cells also take part in regulating innate immune responses during the early phases of infection [14]. A review by Tong Hong and Fu Yangxin entitled "A new role for $\mathrm{T}$ cells in dampening innate inflammatory responses" [15] provides us with a new perspective on T cells. This review cites evidence suggesting that conventional $\mathrm{T}$ cells temper acute innate inflammation, and elaborates upon the recent finding that effector and memory $\mathrm{T}$ cells dampen the hyperactive inflammasome during the late phase of the primary response or upon secondary challenge. These unexpected results suggest that $\mathrm{T}$ cells within the adaptive immune arm regulate innate responses; thus, shedding new light on the evolution and interplay between the innate and adaptive immune systems [16]. Further understanding of the underlying mechanisms will facilitate the clinical diagnosis and treatment of various infectious and inflammatory diseases.

Mice with a reconstituted human immune system, and other human target organs, are very important for research into human-tropic pathogens. As such, a number of human-mouse chimeric models (incorporating human immune cells) have been developed over the past 20 years, but with only limited success. In the final review in this series entitled "Current humanized mouse models for studying human immunology and HIV-1 immuno-pathogenesis", Zhang et al. [17] summarize the current understanding of HIV-1 immunopathogenesis in human patients and SIV-infected primate models. The authors review recent progress in the development of humanized mouse models with a functional human immune system, particularly the recent progress in the development of immunodeficient mice that carry a defective gamma-C gene.

Alongside the development of the national economy and other sciences in China, immunology has developed rapidly over the past ten years. Compared with the limited amount prior to this, immunology research in China is now both extensive and comprehensive, covering all aspects from basic theory to clinical research, and the development and application of immunological techniques. Many excellent Chinese immunologists have emerged over recent years and have produced remarkable work in the field, including the structural basis and mechanisms of immune recognition, immunological therapy, influenza, and inflammasomes. The work on Toll-like receptors, which mediate both innate and adaptive immune responses by recognizing molecules expressed by invading pathogens and eliciting immune responses, has greatly enhanced our understanding of inflammation $[18,19]$. Studies of influenza have also helped the fight against the threat of flu [20-23]. The development of monoclonal antibody techniques has facilitated the development of modern therapeutics for related diseases [24-26] and research on HIV has brought new opportunities to cure AIDS [27,28].

When we look at the overall standard of immunology research in China, there is still a gap when compared with that in developed countries, mainly due to a lack of originality and innovation. However, constantly increasing input and increased attention means that immunology in China should experience a leapfrogging in terms of development in the near future. We hope that this special focus on immunology in this issue of SCIENCE CHINA Life Sciences will help to achieve this.

1 Wang Y Y, Zhang P, Liu Y F, et al. TRAF-mediated regulation of immune and inflammatory responses. Sci China Life Sci, 2010, 53: 159-168

2 Liu Y, Chen G Y, Zheng P, et al. On self-nonself discrimination in pattern recognition. Sci China Life Sci, 2010, 53: 169-171

3 Liu Y, Zheng P. CD24: A genetic checkpoint in T cell homeostasis 
and autoimmune diseases. Trends Immunol, 2007, 28: 315-320

4 Chen G Y, Tang J, Zheng P, et al. CD24 and siglec-10 selectively repress tissue damage-induced immune responses. Science, 2009, 323: 1722-1725

5 Banchereau J, Steinman R M. Dendritic cells and the control of immunity. Nature, 1998, 392: 245-252

6 Siegal F P, Kadowaki N, Shodell M, et al. The nature of the principal type 1 interferon-producing cells in human blood. Science, 1999, 284: 1835-1837

7 Liu Y-J. IPC: Professional type 1 interferon-producing cells and plasmacytoid dendritic cell precursors. Annual Rev Immunol, 2005, 23: 275-306

8 Tang F, Du Q M, Liu Y J. Plasmacytoid dendritic cells in antiviral immunity and autoimmunity. Sci China Life Sci, 2010, 53: 172-182

9 Gilliet M, Cao W, Liu Y J. Plasmacytoid dendritic cells: Sensing nucleic acids in viral infection and autoimmune diseases. Nat Rev Immunol, 2008, 8: 594-606

10 Charles J, Chaperot L, Salameire D, et al. Plasmacytoid dendritic cells and dermatological disorders: Focus on their role in autoimmunity and cancer. Eur J Dermatol, 2010, 20: 16-23

11 Wang S D, Chen L P. Structural immunology of costimualtory and coinhibitory molecules. Sci China Life Sci, 2010, 53: 183-189

12 Carreno B M, Collins M. The B7 family of ligands and its receptors: new pathways for costimulation and inhibition of immune responses. Annu Rev Immunol, 2002, 20: 29-53

13 Dong H, Zhu G, Tamada K, et al. B7-H1, a third member of the B7 family, co-stimulates T-cell proliferation and interleukin-10 secretion. Nat Med, 1999, 5: 1365-1369

14 Kim K D, Zhao J, Yang X, et al. Adaptive immune cells temper initial innate responses. Nat Med, 2007, 13: 1248-1252

15 Tang H, Fu Y X. A new role for T cells in dampening innate inflammatory responses. Sci China Life Sci, 2010, 53: 190-194

16 Palm N W, Medzhitov R. Not so fast: Adaptive suppression of innate immunity. Nat Med, 2007, 13: 1142-1144

17 Zhang L G, Meissner E, Chen J Z, et al. Current humanized mouse models for studying human immunology and HIV-1 immuno-pathogenesis. Sci China Life Sci, 2010, 53: 195-203

18 Sun Bing, Han D S. Negative regulation of Toll-like receptors signaling pathways. Prog Biochem Biophys, 2009, 36: 1516-1522

19 Zhu X M, Yao Y M, Sheng Z Y. Inflammasome and inflammatory response. Prog Biochem Biophys, 2010, 37: 129-137

20 Wang D C. Challenges from epigenetics and avian influenza virus. Prog Biochem Biophys, 2010, 37: 1047-1050

21 Zhou K, He H X. Avian influenza and swine influenza: New understanding of crossing species barrier. Prog Biochem Biophys, 2009, 36: 523-529

22 Sun L, Liu D B, Yang Y D, et al. Regulation of antiviral innate immune responses by human coronavirus. Prog Biochem Biophys, 2010, 37: 239-244

23 Wang G-F, Li K S. Origins and views of the 2009 A/H1N1 influenza pandemic. Prog Biochem Biophys, 2009, 36: 945-949

24 Li Y T, Wu W B, Hong Y, et al. Purification of hCLEC-2 recombinant protein in engineering bacteria and preparation and identification of its antibody. Prog Biochem Biophys, 2009, 36: 1012-1018

25 Chen H X, Zhang X Y, Chen C, et al. Progress on chicken monoclonal antibody technology. Prog Biochem Biophys, 2010, 37: 358-363

26 Pei X H, Chi S, Wang J T, et al. Generation and application of active recombinant mouse tissue factorand its function-blocking monoclonal antibody. Prog Biochem Biophys, 2009, 36: 601-607

27 An Q X, Lei Y F, Yang J, et al. Inhibition of HIV-1 infection by CCR5Delta32 protein expressed in human PBMCs. Prog Biochem Biophys, 2009, 36: 1141-1145

28 He H Q, Hu J P, Liu B, et al. Activity, Solubility comparison and molecular dynamics simulation analysis of wild type and F185K mutant type HIV-1 integrase catalytic domain. Prog Biochem Biophys, 2009, 36: 1146-1153

Open Access This article is distributed under the terms of the Creative Commons Attribution License which permits any use, distribution, and reproduction in any medium, provided the original author(s) and source are credited. 